\title{
Efficacy of adjunctive use of diacerein local delivery in the management of moderate chronic periodontitis: a double-blind randomized controlled trial
}

\author{
Rahma Omar Ahmed ${ }^{1}$, Farid Badria², Medhat EL-daker ${ }^{3}$, Omar Hasan Khashaba ${ }^{4}$
}

${ }^{1}$ M.Sc., Department of Oral Medicine, Periodontology Diagnosis and Oral Radiology, Faculty of Dentistry, University of Mansoura, Mansoura, Egypt

${ }^{2}$ Ph.D., Professor, Department of Pharmacognosy, Faculty of Pharmacy, University of Mansoura, Mansoura, Egypt

${ }^{3}$ Ph.D., Professor, Department of Microbiology and Immunology, Faculty of Medicine, University of Mansoura, Mansoura, Egypt

${ }^{4}$ PhD., Professor, Department of Oral Medicine, Periodontology Diagnosis and Oral Radiology, Faculty of Dentistry, University of Mansoura, Mansoura, Egypt

\section{Type of article: Original}

\begin{abstract}
Background: Chronic periodontitis is a common public health concern characterized by progressive gingival inflammation.

Objective: This study assessed the clinical and laboratory effect of diacerein as adjunctive therapy to scaling and root planing (SRP) in the therapeutic management of moderate chronic periodontitis.

Methods: This double-blind randomized controlled trial was conducted on thirty patients suffering from moderate chronic periodontitis at the dental clinic in the Faculty of Dentistry (University of Mansoura, Egypt) between March-October 2018. The participants were randomly allocated into two groups of fifteen subjects each. The control and study group received SRP and diacerein was administered to the study group in the form of a gelformula applied to the periodontal pocket for 6 weeks. The clinical periodontal parameters assessed pre- and posttreatment were plaque index (PI), gingival index (GI), probing depth, and clinical attachment level (CAL). Samples of gingival crevicular fluid (GCF) were also collected at baseline and six weeks after the initiation of the treatment for IL-1ß assessment using ELISA. Clinical parameters collected were compared between and after treatment by descriptive and inferential statistical analysis using IBMC SPSS $\odot$ Statistics version 20.

Results: Age and gender were not significantly different between the two groups. There was a significant decrease in the median values of clinical indices post-treatment compared to pre-treatment in the study group (test significance $=23.07 ; p<0.001$ ). Interestingly, there was a significant decrease in IL- $\beta 1$ after treatment in both groups, with a more significant decrease in the study group compared to the control group $(\mathrm{p}=0.0001)$.

Conclusion: Diacerein is an alternative and promising therapeutic option for the management and control of chronic periodontitis.

Clinical Trial Registration: The trial has been registered at http://www.clinicaltrials.in.th with registration ID:

TCTR20190930001.

Funding: No funds or financial support, governmental or non-governmental, was received

Keywords: Periodontitis; Chronic periodontitis; Scaling and root planning; Diacerein; IL- $\beta 1$
\end{abstract}

\section{Abbreviations / Acronyms:}

CAL: Clinical Attachment Level; CONSORT: Consolidated Standards of Reporting Trials; CP: Chronic Periodontitis; GCF: Gingival Crevicular Fluid; GI: Gingival Index; NSAID: Nonsteroidal anti-inflammatory Drug; OSDI: Ocular Surface Disease Index; PPD: Probing Pocket Depth; PI: Plaque Index; SRP: Scaling and Root Planing

\section{Corresponding author:}

Rahma Omar Ahmed. Department of Oral Medicine, Periodontology Diagnosis and Oral Radiology, Faculty of Dentistry, University of Mansoura, Mansoura, Egypt. Tel: +201158911860, E-mail: r.o.ahmed@outlook.com Received: September 09, 2020, Accepted: November 12, 2020, Published: December 2020 iThenticate screening: October 21, 2020, English editing: November 19, 2020, Quality control: December 02, 2020 Ethics approval: Medical research ethical committee of the Faculty of Dentistry, University of Mansoura, Egypt. (C) 2020 The Authors. This is an open access article under the terms of the Creative Commons Attribution-NonCommercialNoDerivs License, which permits use and distribution in any medium, provided the original work is properly cited, the use is non-commercial and no modifications or adaptations are made. 
Note: This study has followed the CONSORT Statement, which is an evidence-based, minimum set of recommendations for reporting randomized trials. It offers a standard way for authors to prepare reports of trial findings, facilitating their complete and transparent reporting, and aiding their critical appraisal and interpretation.

\section{Introduction}

Periodontal diseases are common inflammatory disorders associated with opportunistic organisms and dental biofilm formation causing gum retrogression, soft tissue damage, weakness of the bone and eventual osteoporosis (1). Periodontitis affects all age groups and can be classified based on the severity and physiological response into necrotizing periodontitis, periodontitis as a direct manifestation of systemic diseases and periodontitis (include both chronic and aggressive periodontitis) $(2,3)$. Chronic periodontitis is a frequent form of the disease characterized by the low-moderate progression that may affect extend sites (localized and generalized types), however, classification of the progressive lesions in patients has been extensively revised recently $(4,5)$.

Periodontal diseases trigger innate and adaptive immunity involving the release of numerous cytokines and inflammatory mediators (6), such as interleukin IL-1 $\beta$, tumour necrosis prostaglandins (PGE2), IFN $\gamma$, IL-6 and IL-8, major periodontal inflammatory mediators associated with T-helper 1 and T-helper 17 cell phenotypes expressed at high levels in gingival tissues from patients with chronic periodontitis $(7,8)$. These play important roles in bone and collagen destruction through degradation of connective tissues and osteoclast activation $(5,9)$ and are controlled by various regulatory systems and components of the immune system (1).

Regulation and control of the host responses are largely adopted in the treatment of periodontal conditions $(10,11)$, with the local delivery system being the preferred effective route used with scaling and root planing (SRP) and systemic therapy for the management of chronic periodontal conditions (12). This therapeutic intervention applies various anti-inflammatory and antibiotic drugs (e.g. Nonsteroidal anti-inflammatory drug (NSAIDs)), providing better treatment outcomes and less undesirable effects (13). Diacerein, an anthraquinone symptomatic drug, provides high permeability, low solubility and is typically prescribed to treat osteoarthritis, was recently proposed as a potential candidate for the treatment of periodontal diseases $(14,15)$. A recent study from Egypt by Zaki et al. investigated the effect of diacerein in vitro revealing promising therapeutic advantages as a potent anti-inflammatory drug in the management of periodontitis, particularly in the developing regions (16). Therefore, the current study assessed the clinical and laboratory effects of diacerein as adjunctive therapeutic therapy in the management of moderate chronic periodontitis.

\section{Material and Methods}

\subsection{Design of study and selection criteria}

This double-blind randomized controlled trial was conducted in the Dentistry clinic of the Faculty of Dentistry (University of Mansoura, Egypt) between March-October 2018. Patients were included based on specific clinical and diagnostic criteria of moderate chronic periodontitis (5) and categorized as follows:

\subsubsection{Inclusion Criteria}

1) Gender: Both

2) Age Limit (years): Minimum $=35$, and Maximum $=45$

3) Probing depth $3-4 \mathrm{~mm}$ in at least three teeth

4) Attachment loss of $\geq 3 \mathrm{~mm}$

5) A minimum of 20 teeth

6) OSDI (Ocular Surface Disease Index) $>13$

7) Corneal staining score $\geq 3$

\subsubsection{Exclusion Criteria}

1) Allergic to diacerein

2) Use of cyclosporin within 30 days

3) Eyelid problem

4) Change artificial tear while in the study

5) Periodontal treatments or were given antibiotic drugs within the last 3 months before the study

6) Pregnancy or breastfeeding, systemic diseases (including osteoarthritis) and smoking habits. 


\subsection{Randomization and Outcome}

Thirty patients with moderate chronic periodontitis $(n=30)$ were enrolled in the study and divided into two groups, study and control group, each containing fifteen subjects $(n=15)$ (Figure 1). Patients were included based on their availability and willingness to participate, provide accurate information and follow the study guidelines. Participants were also asked to fill in a designated questionnaire which included generic and clinical information for accuracy and assurance of quality. The questionnaire and clinical form were designed to obtain full details throughout the study for further statistical analyses based on recently published data and a local understanding of the culture and habits of the population in Mansoura city. Initial treatment with full-mouth supragingival and subgingival SRP under local anaesthesia using an ultrasonic scaler and hand instrument was performed weekly.

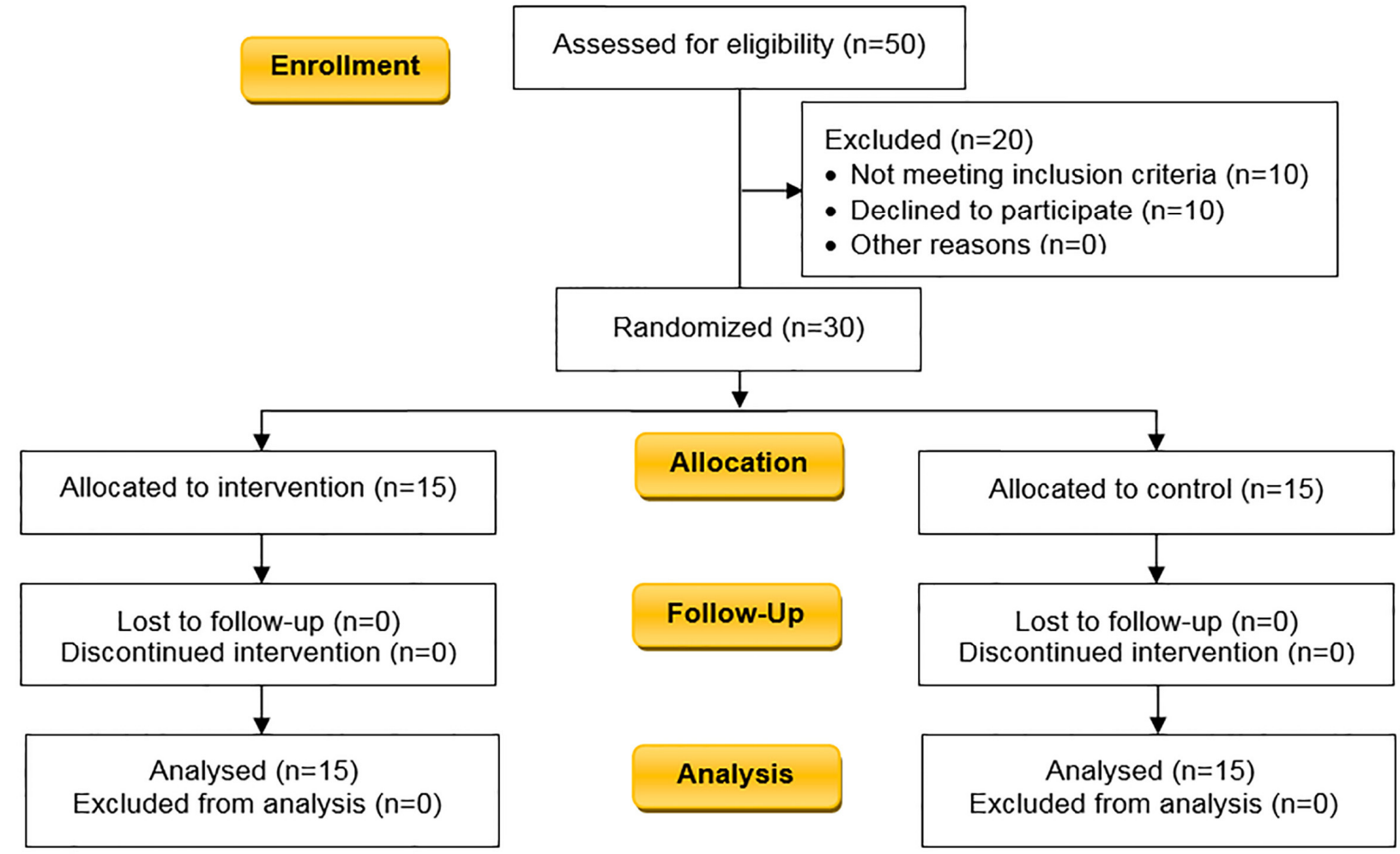

Figure 1. CONSORT 2010 Flow Diagram of the study

\subsection{Gel preparation and local drug delivery of diacerein}

Diacerein capsules $(500 \mathrm{mg}$ hard gelatin) (EvaPharma) $(1.2 \% \mathrm{w} / \mathrm{w})$ were dissolved in both propylene glycol and glycerin as described by Ahmad et al. (17). Gels were then packed in wide glass jars covered with aluminium foil and tightly capped with plastic lids and kept in a cool dark place until use (18). The diacerein gel was locally delivered into the pocket using a syringe with blunt cannula once per week for 6 weeks.

\subsection{Treatment protocol (study group)}

Patients in the study group were given approximately $4 \mathrm{ml}$ of diacerein gel using a local delivery technique into the periodontal pocket via a syringe with a blunt cannula. Patients were subjected to the experimental treatment and followed for consecutive six weeks. They were provided with detailed information on oral hygiene and asked to refrain from chewing any food materials or brushing close to the treated area, not to rinse with water nor drink anything at least 30 minutes after treatment, and not to use any interdental aids. Patients were also instructed to follow self-performed oral hygiene including brushing regularly daily. Anti-inflammatory and/or antibiotics agents were not given or prescribed after treatment. Also, patients were closely followed for any gingival deposits and adverse reactions at each visit.

\subsection{Assessment of clinical parameters and collection of samples}

The case history of each patient was collected and clinically examined for color, contour, as well as the normal size and texture of the gingival tissue. Clinical and periodontal parameters were collected following typical procedures 
including plaque index (PI), gingival index (GI), probing pocket depth (PPD) for each patient. Periodontal indices of the control group were measured pre and post six weeks of treatment. Periodontal indices of the study group were also measured throughout the six weeks of treatment. Samples of GCF were also collected at baseline as well as post-treatment to assess IL-1ß.

\subsection{Laboratory analysis of $I L-1 \beta$}

The IL-1ß ELISA was a sandwich-ELISA method (Elabscience Biotechnology Co Ltd, Wuhan, China). Initially, GCF was collected from each pocket using sterile paper (Periopaper) strips at baseline and post-treatment. The strips were inserted into the pocket (gingival crevice) until light resistance was felt, then held for $30 \mathrm{~s}$ before placing into sterile Eppendorf tubes and stored immediately at $-80^{\circ} \mathrm{C}$ for further analysis. The ELISA was performed in duplicate for each fluid-sample.

\subsection{Statistical analysis}

Data was input into Microsoft Excel and descriptive and inferential statistical analyses performed using IBM $C$ SPSS(C) Statistics version 20 (IBM(C) Corp., Armonk, NY, USA). Description statistical analysis of the qualitative clinical data was performed, with Chi-square and paired t-tests used for categorical variables and comparison purposes between both groups. Results were considered significant at $\mathrm{p}<0.001$.

\subsection{Ethics of Research}

The experimental study was approved by the Medical research ethical committee of the Faculty of Dentistry, University of Mansoura, Egypt. The purpose of the study and the benefits of their participation was explained to all participants before the study, and written informed consent was obtained. The study obtained clinical trial registration (ID: TCTR20190930001) at http:/www.clinicaltrials.in.th/.

\section{Results}

The mean age in the study and control groups was 41.2 and 38.8 years, respectively (Table 1 ), with more females in the study group than the control group.

Table 1. Sociodemographic characteristics of participants

\begin{tabular}{|l|l|l|l|l|}
\hline Variables & Study Group & Control Group & Test Significance \\
\hline \multicolumn{2}{|l|}{ Age $($ Mean \pm SD; years) } & $41.2 \pm 2.93$ & $38.8 \pm 3.8$ & $\mathrm{t}=1.94(\mathrm{p}=0.06)^{*}$ \\
\hline $\begin{array}{l}\text { Gender; N } \\
(\%)\end{array}$ & Male & $1(6.7)$ & $3(20.0)$ & FET $(\mathrm{p}=0.59)^{* *}$ \\
\cline { 2 - 5 } & Female & $14(93.3)$ & $12(80.0)$ & \\
\hline
\end{tabular}

* t-test; **Fischer Exact Test

The median values of clinical indices were compared pre- and post-treatment showing a significant decrease in the study group compared to the control group (Table 2). Also, there was a statistically significant decrease in the median values of the clinical indices post-treatment compared to pre-treatment in the control group (Table 2). In addition, there was a significant decrease in the level of IL- $\beta 1$ in patients before and after therapy with a more significant decrease in the study group (61.9\%) compared to the control group $(42.9 \%)(p=0.0001)$ (Table 3$)$.

Table 2. Median of clinical indices before and after treatment among the study group

\begin{tabular}{|c|c|c|c|c|c|c|}
\hline \multirow[t]{2}{*}{ Variable } & \multicolumn{3}{|l|}{ Study group } & \multicolumn{3}{|l|}{ Control group } \\
\hline & $\begin{array}{l}\text { Before } \\
\text { treatment }\end{array}$ & $\begin{array}{l}\text { After } \\
\text { treatment }\end{array}$ & $\begin{array}{l}\text { Test } \\
\text { Significance }\end{array}$ & $\begin{array}{l}\text { Before } \\
\text { treatment }\end{array}$ & After treatment & $\begin{array}{l}\text { Test } \\
\text { Significance }\end{array}$ \\
\hline PI & $\begin{array}{l}2.83(1.95- \\
3.0)\end{array}$ & $\begin{array}{l}0.33(0.0- \\
0.83)\end{array}$ & $\begin{array}{l}Z=3.41 \\
\left(p=0.001^{\dagger}\right)\end{array}$ & $\begin{array}{l}2.66(1.91- \\
3.0)\end{array}$ & $1.5(0.5-2.5)$ & $\begin{array}{l}Z=3.43 \\
\left(p=0.001^{\dagger}\right)\end{array}$ \\
\hline GI & $\begin{array}{l}2.66(2.16- \\
3.0)\end{array}$ & $\begin{array}{l}0.33(0.0- \\
1.33)\end{array}$ & $\begin{array}{l}Z=3.4 \\
\left(p=0.001^{\dagger}\right)\end{array}$ & $\begin{array}{l}2.66(1.91- \\
3.0)\end{array}$ & $1.85(1.0-2.33)$ & $\begin{array}{l}\mathrm{Z}=3.42 \\
\left(\mathrm{p}=0.001^{\dagger}\right)\end{array}$ \\
\hline GBI $\%$ & $\begin{array}{l}44.33(36.0- \\
50.0)\end{array}$ & $\begin{array}{l}8.33(0.0- \\
33.3)\end{array}$ & $\begin{array}{l}Z=3.41 \\
\left(p=0.001^{\dagger}\right)\end{array}$ & $\begin{array}{l}44.33(36.0- \\
50.0)\end{array}$ & $\begin{array}{l}27.66(8.33- \\
40.66)\end{array}$ & $\begin{array}{l}\mathrm{Z}=3.42 \\
\left(\mathrm{p}=0.001^{\dagger}\right)\end{array}$ \\
\hline PD & $\begin{array}{l}3.71(2.63- \\
5.02)\end{array}$ & $\begin{array}{l}1.01(0.01- \\
1.20)\end{array}$ & $\begin{array}{l}Z=3.40 \\
\left(p=0.001^{\dagger}\right)\end{array}$ & $\begin{array}{l}3.61(2.36- \\
4.64)\end{array}$ & $1.22(1.0-2.84)$ & $\begin{array}{l}Z=3.41 \\
\left(p=0.001^{\dagger}\right)\end{array}$ \\
\hline $\mathrm{CL}$ & $\begin{array}{l}3.71(2.63- \\
5.02)\end{array}$ & $\begin{array}{l}1.01(0.01- \\
1.20)\end{array}$ & $\begin{array}{l}\mathrm{Z}=3.41 \\
\left(\mathrm{p}=0.001^{\dagger}\right)\end{array}$ & $\begin{array}{l}3.61(2.36- \\
4.64)\end{array}$ & $1.22(1.0-2.84)$ & $\begin{array}{l}\mathrm{Z}=3.40 \\
\left(\mathrm{p}=0.001^{\dagger}\right)\end{array}$ \\
\hline
\end{tabular}

Median* estimates are presented as minimum to maximum values; $\uparrow$ : statistically significant $(\mathrm{p}<0.05)$; z: Wilcoxon signed rank test; p: probability. 
Table 3. The level of IL- $\beta 1$ change between before and after treatment among study \& control groups

\begin{tabular}{|l|l|l|l|l|l|l|}
\hline \multirow{2}{*}{ IL- $\beta 1$} & \multicolumn{3}{|l|}{ Study Group } & \multicolumn{3}{l|}{ Control Group } \\
\cline { 2 - 7 } & Before treatment & After treatment & Test Sig. & Before treatment & After treatment & Test Sig. \\
\hline Mean & $34.2 \pm 5.40$ & $13.02 \pm 3.54$ & $\mathrm{t}=23.07$ & $31.81 \pm 5.72$ & $18.15 \pm 3.05$ & $\mathrm{t}=12.23$ \\
\pm SD & & & $\mathrm{p}<0.001^{\dagger}$ & & & $\mathrm{p}<0.001^{\dagger}$ \\
\hline
\end{tabular}

t: paired t test value; SD: Standard deviation; $\mathrm{p}$ : probability; $\uparrow:$ statistically significant

\section{Discussion}

Chronic periodontitis is a common periodontal condition mainly affecting adults characterized by a progressive gingival inflammation (19). Various plaque-causing organisms are responsible for the initiation and progression of the disease leading to major inflammatory reactions $(9,20)$. Chronic periodontitis mainly affects adults and is associated with various systemic and non-systemic conditions (e.g. psychological stress, metabolic, diabetes, obesity, cardiovascular diseases), and has several putative risk factors (e.g. ageing, gender, smoking, metabolic syndromes, diabetes, alcohol, osteoporosis, and genetic factors) (21).

In the current study, there was no significant difference in age between the study and control group, however, recent studies have shown an association between age and the severity of chronic periodontitis. A recent report revealed that patients aged 20-44 years old had a slight increase in the prevalence of mild chronic periodontitis and that the severity tends to increase with age. Such an increase is linked to the accumulative effect of age as well as the continuous and prolonged exposure to risk factors, including microbial challenge, but not entirely due to ageing itself (22). A recent in vivo study demonstrated that the ageing process produces better immunity, therefore the ageing process may not entirely cause the increased prevalence of periodontal diseases (23). Generally, males are more at risk of periodontal diseases and the age cumulative effect rather than age itself is linked to the severity of chronic periodontitis (23). A recent study reported that males particularly with a low level of education and older than 30 years were more likely to be affected by periodontitis (24). In our study, there were more females in both groups but no significant difference between groups, which might be attributed to the low number of participants included in this study. A recent study involving 1000 female patients aged 25-75 years old revealed that half of the investigated females suffered from periodontal disease, with factors such as age and occupation being potential risks for chronic periodontitis (25).

Oral hygiene is a major contributor to the progression and treatment outcome of periodontal diseases (26). Nonsurgical SRP intervention can effectively control periodontal conditions and maintain A healthy periodontium (20). Such therapeutic interventions improve the hygiene status of the gingiva, clean the root surface, thereby avoiding recolonization of plaque pathogens and recurrent periodontitis. A study by Schatzle et al. revealed that almost quarter of the investigated individuals did not develop chronic periodontitis and that keeping a healthy oral periodontal status may significantly prevent the development and progression of periodontal disease (27). In the current study, there was no significant difference in the clinical indices before treatment between both groups. However, there was a significant decrease in the clinic indices post-treatment compared to pre-treatment in both groups. The non-surgical SRP option is important for the management and control of chronic periodontitis, hence, is frequently used along with mechanical treatment using antimicrobial and anti-inflammatory drugs (28).

The local drug delivery system into the periodontal pocket is an extension treatment to SRP and is an effective and non-invasive therapeutic route that uses various agents such as antimicrobials (e.g. NSAIDs) and anti-inflammatory agents providing significant therapeutic advantages (10). Diacerein is an anthraquinone derivative, originating from plants with anti-inflammatory and analgesic properties, however, it may cause uncomfortable GI irritation. The use of gel and other formulas can provide an easy, comfortable and efficient route of diacerein delivery to overcome GI undesirable reactions (29). In addition, all included participants were between 35-45 years of healthy-stable status which provide further persistent and endurance against any foreign materials including medications.

Certain immune and inflammatory biomarkers are associated with the severity of chronic periodontitis, particularly IL-1 $\beta$ (30). In the current study, there was no significant difference in the level of IL- $\beta 1$ between both groups, however, there was a significant decrease in the study group post-treatment. This is an important finding that potentially indicates the significant effect of diacerein on reducing the level of IL- $\beta 1$ resulting in a better inflammatory resolution. This is linked to the mode of action of this drug which suppresses IL-1 and related downstream reactions by inhibiting the IL-1-converting enzyme (14). These findings are in line with a recent experimental trial by Zaki et al. which investigated the effect of diacerein on laboratory experimental animals 
revealing a significant decrease of IL- $\beta 1$ (16). Taken together, these findings suggest that diacerein may significantly contribute to the management of Chronic Periodontitis (CP).

The host reactions to periodontal pathogens are complex, involving the release of both local and systemic cytokines, which are produced by the gingival epithelium and inflammatory cells resulting in uncontrolled inflammatory reactions affecting the tooth-supporting structures (1). Of these, IL-1 $\beta$, IFN $\gamma$, and TNF- $\alpha$ are the major inflammatory biomarkers for periodontal diseases and associated with the induction and severity of chronic periodontitis (30). These can be found in serum, plasma, GCF, and saliva of chronic periodontitis and can be used as markers to distinguish and discriminate healthy status from periodontitis cases (31). IL-1 $\beta$ is an important cytokine in the regulation of immune responses in periodontium as well as a major player in the pathogenesis of chronic periodontal diseases (32). Recent studies have documented the association between the clinical measures of periodontal diseases and salivary levels of IL-1 $\beta$, with a high level of IL-1 $\beta$ associated with increased alveolar bone loss and bone resorption (33). In addition, IL-1 $\beta$ is a downstream regulator of tissue destruction in periodontitis, such as osteoclastic bone resorption, degeneration of collagen and matrix metalloproteinases.

\section{Study strengths and limitations}

To the best of our knowledge, this is the first study to examine the application of diacerein as adjuvant therapy in the management of chronic periodontitis. Nonetheless, the study has some limitations. First, the participants may have provided unreliable information regarding their recent history of smoking or drug use. This was difficult to achieve due to local cultural customs which negatively affect research medical programme, particularly trials. Also, due to limited resources available only the level of IL-1 $\beta$ was evaluated. Nevertheless, the significant reduction in IL-1 $\beta$ and oral improvement in the periodontal health of the study group compared to the control group indicates the potential and promising therapeutic route of diacerein in the management of chronic periodontitis.

\section{Conclusions}

This study investigated the therapeutic effect of the local delivery of diacerein in the management of chronic periodontitis. Diacerein in combination with non-surgical SRP was effective for the management and control of chronic periodontitis. This study provides essential and novel information for health and medical policymakers, as well as dental practitioners. Further investigations are required to understand the extent of the diacerein effect on other immune elements that are reportedly associated with periodontitis.

\section{Acknowledgments:}

This work was extracted from a postgraduate thesis performed at the Faculty of Dentistry, University of Mansoura, Egypt. No funds or financial support, governmental or non-governmental, was received. The corresponding author would like to extend her heartfelt appreciation for the kind support of Prof. Omar Hasan Khashaba.

\section{Conflict of Interest:}

There is no conflict of interest to be declared.

\section{Authors' contributions:}

Conception or design of the work: ROA, ME, OHK; Acquisition of data: ROA; Analysis or interpretation of data: ROA, FB; Drafting the manuscript: ROA, OHK; Revising the manuscript: All authors; Accountable for all aspects of the work: All authors. All authors read and approved the final manuscript.

\section{References:}

1) Silva, N. et al. Host response mechanisms in periodontal diseases. J Appl Oral Sci, 2015 May-Jun; 23(3):329-55. doi: 10.1590/1678-775720140259, PMid:26221929, PMCid: PMC4510669

2) Tonetti, M.S., Greenwell, H. and Kornman, K.S. Staging and grading of periodontitis: Framework and proposal of a new classification and case definition. J Periodontol. 2018;89(1):S159-S172. doi: 10.1002/JPER.18-0006, PMid: 29926952

3) López R, Baelum V. Periodontal disease classifications revisited. European Journal of Oral Sciences, 2015;123(6):385-9. doi: 10.1111/eos.12227, PMid: 26505468

4) Wiebe, C.B. and Putnins, E.E.. The periodontal disease classification system of the American Academy of Periodontology--an update. Journal (Canadian Dental Association), 2000;66(11):594-7. 
5) American Academy of Periodontology Task Force Report on the Update to the 1999 Classification of Periodontal Diseases and Conditions. Journal of Periodontology. 2015;86(7):835-8. doi: 10.1902/jop.2015.157001, PMid: 26125117

6) Bartold, P.M., Cantley, M.D. and Haynes, DR. Mechanisms and control of pathologic bone loss in periodontitis. Periodontology 2000, 2010;53:55-69. doi: 10.1111/j.1600-0757.2010.00347.x, PMid: 20403105

7) Cochran, D.L. Inflammation and bone loss in periodontal disease. Journal of Periodontology, 2008;79(8 Suppl):1569-76. doi: 10.1902/jop.2008.080233, PMid: 18673012

8) Stashenko, P., Van, D.T., Tully, P., Kent, R., Sonis, S. and Tanner, A.C. Inflammation and genetic risk indicators for early periodontitis in adults. Journal of Periodontology, 2011;82(4):588-96. doi: 10.1902/jop.2010.100443, PMid:21054222, PMCid:PMC3299480

9) Pihlstrom, B.L., Michalowicz, B.S. and Johnson, N.W. Periodontal diseases. Lancet, 2005;19(9499):180920. doi: 10.1016/S0140-6736(05)67728-8

10) Hosn, K.N., Jefferson, M.M., Leding, C., Shokouh-Amiri, S. and Thomas, E.L. Inhibitors of bacterial protease enzymes for periodontal therapy. Clinical and Experimental Dental Research, 2015;1(1):18-25. doi: 10.1002/cre2.4, PMid:29744136, PMCid:PMC5839262

11) Anonymous. Treatment of Plaque-induced Gingivitis, Chronic Periodontitis, and Other Clinical Conditions. Pediatric Dentistry. 2017;39(6):445-54.

12) Nair, S.C. and Anoop, K.R. Intraperiodontal pocket: An ideal route for local antimicrobial drug delivery. Journal of Advanced Pharmaceutical Technology and Research, 2012;3(1):9-15.

13) Seydanur Dengizek, E., Serkan, D., Abubekir, E., Aysun Bay, K., Onder, O. and Arife, C. Evaluating clinical and laboratory effects of ozone in non-surgical periodontal treatment: a randomized controlled trial. J Appl Oral Sci, 2019 Jan 14;27:e20180108. doi: 10.1590/1678-7757-2018-0108, PMid: 30673028, PMCid: PMC6438664

14) Huang, R. Y., Lu, S. H., Su, K. W., Chen, J. K., Fang, W. H., Liao, W. N., Chen, S. Y. and Shieh, Y. S. Diacerein: a potential therapeutic drug for periodontal disease. Medical Hypotheses. 2012;79(2):165-7. doi: 10.1016/j.mehy.2012.04.024, PMid: 22583561

15) Panova, E. and Jones, G. Benefit-risk assessment of diacerein in the treatment of osteoarthritis. Drug Safety, 2015;38(3):245-52. doi: 10.1007/s40264-015-0266-z, PMid: 25652235

16) Zaki, B.M., Mahmoud, E.A. and Aly, A.A. Diacerein: A potential therapeutic drug for the management of experimental periodontitis in rats. Electronic Physician, 2015;7(5):1290-5.

17) Ahmad, N., Lonardo, E.C., Patel, K.J., Lin, S.Y., Wearley, L.L., Matheson, J.N. and Wiita, B. Novel methods of treating local and bacterial infections. US Patents 200301302251A, 2003

18) Kumar, L. and Verma, R. In vitro evaluation of topical gel prepared using natural polymer. International Journal of Drug Delivery, 2010;2:58-63. doi: 10.5138/ijdd.2010.0975.0215.02012

19) Sima, C. and Van Dyke, T.E. Therapeutic Targets for Management of Periodontitis and Diabetes. Current Pharmaceutical Design, 2016;22(15):2216-37. doi: 10.2174/1381612822666160216150338, PMid: 26881443

20) Baek, K., Ji, S. and Choi, Y. Complex Intratissue Microbiota Forms Biofilms in Periodontal Lesions. Journal of Dental Research, 2018;97(2):192-200. doi: 10.1177/0022034517732754, PMid: 28945499, PMCid: PMC6429573

21) AlJehani, Y.A. Risk factors of periodontal disease: review of the literature. International Journal of Dentistry, 2014;2014:182513. doi: 10.1155/2014/182513, PMid: 24963294, PMCid: PMC4055151

22) Wu, Y., Dong, G., Xiao, W., Xiao, E., Miao, F., Syverson, A., Missaghian, N., Vafa, R., Cabrera-Ortega, A. A., Rossa, C., Jr, and Graves, D. T. Effect of Aging on Periodontal Inflammation, Microbial Colonization, and Disease Susceptibility. Journal of Dental Research, 2016;95(4):460-6. doi: 10.1177/0022034515625962, PMid: 26762510, PMCid: PMC4802783

23) Petersen, P.E. and Ogawa, H. The global burden of periodontal disease: towards integration with chronic disease prevention and control. Periodontology 2000, 2012;60(1):15-39. doi: 10.1111/j.16000757.2011.00425.x, PMid: 22909104

24) Mendonça, S. A., Teixeira, F. G., Oliveira, K. M., Santos, D. B., Marques, L. M., Amorim, M. M. and Gestinari, R. Study of the association between the interleukin-1 $\beta$ c.3954C $>$ T polymorphism and periodontitis in a population sample from Bahia, Brazil. Contemporary Clinical Dentistry, 2015;6(2):17682. doi: 10.4103/0976-237X.156040, PMid: 26097351, PMCid: PMC4456738

25) Al Qahtani, N.A., Joseph, B., Deepthi, A. and Vijayakumari, B.K. Prevalence of chronic periodontitis and its risk determinants among female patients in the Aseer Region of KSA. Journal of Taibah University 
Medical Sciences, 2017;12(3):241-8. doi: 10.1016/j.jtumed.2016.11.012, PMid: 31435246, PMCid: PMC6694882

26) Lertpimonchai, A., Rattanasiri, S., Arj-Ong Vallibhakara, S., Attia, J. and Thakkinstian, A. The association between oral hygiene and periodontitis: a systematic review and meta-analysis. International Dental Journal, 2017;67(6):332-43. doi: 10.1111/idj.12317, PMid: 28646499, PMCid: PMC5724709

27) Schätzle M. et al. Clinical course of chronic periodontitis. III. Patterns, variations and risks of attachment loss. Journal of Clinical Periodontology, 2003;30(10):909-18. doi: 10.1034/j.1600-051X.2003.00401.x, PMid: 14710771

28) Sanz. I., Alonso, B., Carasol, M., Herrera, D. and Sanz, M. Nonsurgical treatment of periodontitis. Journal of Evidence-Based Dental Practice, 2012;12(3 Suppl):76-86. doi: 10.1016/S1532-3382(12)70019-2

29) Allam, A.N., Hamdallah, S.I. and Abdallah, O.Y. Chitosan-coated diacerein nanosuspensions as a platform for enhancing bioavailability and lowering side effects: preparation, characterization, and ex vivo/in vivo evaluation. International Journal of Nanomedicine. 2017;12:4733-45. doi: 10.2147/IJN.S139706, PMid: 28740381, PMCid: PMC5503500

30) Gomes, F.I., Aragão, M.G., Barbosa, F.C., Bezerra, M.M., de Paulo Teixeira Pinto, V. and Chaves, H.V. Inflammatory Cytokines Interleukin- $\beta$ and Tumour Necrosis Factor- $\alpha$ - Novel Biomarkers for the Detection of Periodontal Diseases: a Literature Review. Journal of Oral and Maxillofacial Research, 2016;7(2):e2. doi: 10.5037/jomr.2016.7202, PMCid: PMC4970502

31) Ebersole, J.L., Nagarajan, R., Akers, D. and Miller, C.S. Targeted salivary biomarkers for discrimination of periodontal health and disease(s). Frontiers in Cellular and Infection Microbiology, 2015;5:62. doi: 10.3389/fcimb.2015.00062, PMid: 26347856, PMCid: PMC4541326

32) Deshpande, N. and Dave, D. Antigen-presenting cells in periodontal disease. Indian Journal of Oral Sciences, 2012;3(2):69-73. doi: 10.4103/0976-6944.106457

33) Miller, C.S., King, C.P. Jr., Langub, M.C., Kryscio, R.J. and Thomas, M.V. Salivary biomarkers of existing periodontal disease: a cross-sectional study. Journal of The American Dental Association, 2006;137(3):322-9. doi: 10.14219/jada.archive.2006.0181, PMid: 16570465 